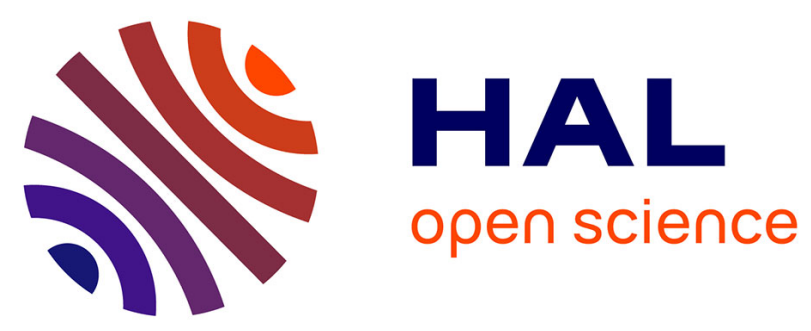

\title{
Dimanche à Miras, lundi à Dipotamia. La frontière albano-grecque dans la région de Bilisht et de Kastoria
}

Michel J. Roux, Gilles de Rapper, Olivier Deslondes

\section{To cite this version:}

Michel J. Roux, Gilles de Rapper, Olivier Deslondes. Dimanche à Miras, lundi à Dipotamia. La frontière albano-grecque dans la région de Bilisht et de Kastoria. Cahiers d'études sur la Méditerranée orientale et le monde turco-iranien, 2000, 29 (1), pp.199-223. 10.3406/cemot.2000.1527 . halshs00258611

\section{HAL Id: halshs-00258611 \\ https://shs.hal.science/halshs-00258611}

Submitted on 11 Apr 2019

HAL is a multi-disciplinary open access archive for the deposit and dissemination of scientific research documents, whether they are published or not. The documents may come from teaching and research institutions in France or abroad, or from public or private research centers.
L'archive ouverte pluridisciplinaire HAL, est destinée au dépôt et à la diffusion de documents scientifiques de niveau recherche, publiés ou non, émanant des établissements d'enseignement et de recherche français ou étrangers, des laboratoires publics ou privés. 


\section{Dimanche à Miras, lundi à Dipotamia. La frontière albano-} grecque dans la région de Bilisht et de Kastoria

\section{Michel Roux, Gilles De Rapper, Olivier Deslondes}

\section{Résumé}

Michel Roux, Gilles de Rapper \& Olivier Deslondes, «Dimanche à Miras, lundi à Dipotamia. La frontière albano-grecque dans la région de Bilisht et de Kastoria», pp 199-222.

L'ouverture de la frontière albano-grecque, vue à travers les relations entre deux villages frontaliers, pourrait faire jouer l'énorme disparité de revenus entre les deux pays en faveur d'un développement économique rapide : les Grecs emploient les Albanais en Grèce, et pourraient les employer en Albanie à condition que la sécurité de leurs investissements soit assurée. Pour le moment, l'instabilité de l'Albanie et la méfiance entre Grecs et Albanais freinent la normalisation des relations, préalable à tout développement. MR, GdR, OD.

\section{Citer ce document / Cite this document :}

Roux Michel, De Rapper Gilles, Deslondes Olivier. Dimanche à Miras, lundi à Dipotamia. La frontière albano-grecque dans la région de Bilisht et de Kastoria. In: CEMOTI, n²9, 2000. L'humanitaire. L'Albanie. pp. 199-224;

doi : https://doi.org/10.3406/cemot.2000.1527

https://www.persee.fr/doc/cemot_0764-9878_2000_num_29_1_1527

Fichier pdf généré le 28/03/2018 
Cahiers d'études sur la Méditerranée

orientale et le monde turco-iranien,

$\mathrm{N}^{\circ} 29$, janvier-juin 2000

\title{
DIMANCHE À MIRAS, LUNDI À DIPOTAMIA LA FRONTIÈRE ALBANO-GRECQUE DANS LA RÉGION DE BILISHT ET DE KASTORIA
}

\author{
Gilles DE RAPPER, Olivier DESLONDES, Michel ROUX
}

\section{De Miras à Dipotamia}

Albanie, dimanche après-midi de septembre 1998. De Bilisht, bourgade de 8000 habitants récemment promue préfecture du district du Devoll (43000 habitants), un vieux taxi Mercedes nous emmène au village de Miras à travers un paysage sans arbre où les petites parcelles de maïs, de trèfle et de haricots, redécoupées dans l'ancien parcellaire socialiste, alternent avec de vastes étendues laissées en pâture au bétail. Des paysans s'affairent à tout cela, d'autres circulent sans hâte, à pied ou en carriole. Une autre Mercedes nous croise à grande allure dans un nuage de poussière. De loin en loin, les rangs de casemates hérité des fantasmes d'Enver Hoxha regardent à l'est les collines décharnées, toutes proches, où court la frontière grecque. Au sud et à l'ouest, le bassin agricole est fermé par les versants du Grammos et de la Morava. La commune de Miras occupe cette partie méridionale du Devoll. Elle y groupe 12000 habitants répartis en une dizaine de localités.

Le chef-lieu est un gros village de 3500 habitants dont l'entrée est défigurée par les ruines des bâtiments coopératifs, saccagés et dépecés par la fureur anticollectiviste de 1991. Chacun empile ici au bord de la route, pour l'expédier, sa récolte de gros oignons, spécialité du cru. La rue principale, creusée d'ornières et bordée de maisons en torchis ou en brique crue, conduit quelques centaines de mètres plus loin à une place centrale très animée. L'ancienne maison de la culture, devenue salle polyvalente, déborde d'une noce joyeuse et bruyante. Nous sommes happés par la fête : il faut entrer, prendre place à la table d'honneur, manger et trinquer au rythme des libations et aux accents d'un orchestre tonitruant qui fait onduler la farandole au milieu des buveurs de raki. "Les Grecs sont riches et leurs routes sont asphaltées, mais ils sont tristes et ne rient jamais", nous expliquera-t-on. "Les Albanais, eux..." Seuls, les mariés arborent le sérieux de circonstance : ne vont-ils pas quitter leurs parents respectifs ? On ne nous laisse partir qu'avec la promesse 
de revenir, ce que nous ferons le surlendemain pour rencontrer les édiles, dans une ambiance cette fois plus contrainte.

Grèce, lundi matin. De Kastoria, préfecture de nomos ${ }^{1}$ de 20000 habitants et capitale grecque de la fourrure, le bus local nous emmène au village frontalier de Dipotamia, voisin de Miras. La route traverse l'openfield céréalier en longeant le cours supérieur du fleuve Aliakmon, puis s'élève sur les contreforts du Grammos. Au creux d'un vallon encaissé dans les collines de grès tendres et de marnes, Dipotamia est le chef-lieu du dème d'Akritès, formé en 1991 par la fusion de trois communes ${ }^{2}$, et auquel le recensement accorde cette année-là 933 habitants.

Ici, en effet, la route est asphaltée, et même ré-asphaltée grâce aux subsides de l'Union Européenne. De grosses pelleteuses creusent les dernières tranchées d'un réseau d'évacuation d'eaux usées. Les maisons sont fraîchement badigeonnées de blanc. La place centrale se veut coquette avec son église, son café, son monument aux morts et sa mairie où flotte le drapeau bleu et blanc. Des effluves de crottin et de fumier annoncent l'activité principale : si Miras cultive les oignons, Dipotamia élève des brebis et des vaches. Pourtant, chênes et châtaigniers prolifêrent au-dessus du village, épargnés par des pratiques d'élevage très différentes de celles de l'Albanie. Les arbres ne font pas défaut, les enfants sont rares ou peu visibles, et les vieux nombreux : quel contraste avec Miras!

Le secrétaire du dème nous accueille avec bonhommie, en compagnie de quelques badauds présents à cette heure matinale. La conversation s'engage à bâtons rompus sur la frontière, l'Albanie, les voisins retrouvés après cinquante ans de séparation sans le moindre contact. Retrouvailles qui ne vont pas sans méfiance. On sursaute en apprenant notre escapade de la veille : "Quelle inconscience, que de risques encourus! Les Albanais sont tous armés et des brigands détroussent les passants : vous pouviez revenir nus. Même ici, ce n'est plus sûr depuis qu'ils franchissent librement la frontière". Sueurs froides rétrospectives. La discussion se transportera au café, ralliera d'autres personnages et s'enrichira de points de vue fort variés, puis, en fin d'après-midi, d'une conclusion sentencieuse : " Que voulez-vous savoir d'autre sur l'Albanie ? demande généreusement l'un de nos interlocuteurs. - Quand y êtes-vous allé ? - Moi ? Jamais !"

\footnotetext{
${ }^{1}$ Equivalent grec du département français.

${ }^{2}$ La réforme en cours de l'organisation territoriale prévoit la fusion des communes rurales en dèmes, collectivités locales ayant sensiblement la taille et l'importance des cantons français. Le dème des Akrites (Akritas) comprend les anciennes communes de Dipotamia (658 hab. en 1991, ex-Revani), Chionato (123 hab., ex-Guerliani) et Komninadès (152 hab., ex-Siaki). L'appellation du dème fait plus que rappeler une position géographique (Akritès signifie confins) : elle signale aussi un front de l'hellénisme.
} 
Voilà les débuts d'une enquête visant à comprendre la signification et le rôle local d'une frontière qui a brutalement ouvert l'un sur l'autre, en 1990, deux Etats très inégalement développés. Les disparités salariales et les complémentarités de ressources pourraient conduire le haut Devoll et la Macédoine grecque occidentale à un développement d'autant plus rapide que ces anciens culs-de-sac sont aujourd'hui directement reliés par l'une des deux "grandes" voies routières entre la Grèce et l'Albanie. Mais ces possibilités sont contrariées par l'instabilité et l'incertitude qui agitent l'Albanie depuis 1997, et par les réflexes de méfiance entre Grecs et Albanais. La mémoire ou les fantasmes collectifs pèsent sur les discours et les comportements, qu'il importe d'analyser en conséquence.

\section{Frontière, peuples et nations}

Jusqu'en 1912 sous administration ottomane, l'Albanie et la Macédoine sont un terrain d'affrontement des ambitions nationales serbe, grecque, bulgare et albanaise. Les Albanais sont les derniers venus dans la compétition pour la formation d'un territoire national : ils ne proclament leur indépendance et ne forment un premier gouvernement provisoire, à Vlorë, que le 28 novembre 1912, pendant que les Etats balkaniques coalisés contre la Turquie sont en train d'envahir et d'occuper ses dernières possessions européennes. Les Grecs s'emparent de l'Épire jusqu'à une ligne Korçë-Gjirokastër, tandis que les Serbes s'attribuent le nord et l'est des régions albanophones, partageant la Macédoine avec les Grecs et les Bulgares sans nul souci des prétentions albanaises à constituer un Etat englobant tous les territoires peuplés peu ou prou d'albanophones jusqu'à une ligne Iannina-Kastoria-Veles-SkopjeMitrovica.

Ces prétentions sont appuyées par l'Italie et l'Autriche, qui souhaitent la création d'un Etat albanais le plus vaste possible entre la Serbie et l'Adriatique. Inversement, Serbes et Grecs ont le soutien des Russes et des Français, soucieux de contenir le camp rival et de réduire l'Albanie naissante à sa plus simple expression territoriale. Le tracé frontalier retenu le 29 juillet 1913 par les puissances réunies à Londres est donc un compromis entre les prétentions territoriales albanaises et les ambitions rivales des Etats constitués, d'où naît une petite Albanie privée notamment du Kosovo. Encore la Grèce et la Serbie reviennent-elles à la charge pendant la première guerre mondiale, n'évacuant qu'en 1921 les territoires indûment occupés.

Mais au moment de sa création, la frontière entre la Grèce et l'Albanie ne sépare pas encore deux populations homogènes et distinctes, identifiables à deux nations. Entre Korçë et Kastoria, la frontière n'est ni linguistique, ni religieuse, ni " ethnographique ", pas plus qu'elle ne répond à un quelconque 
"plébiscite" par lequel les habitants de la région eux-mêmes se seraient décidés sur leur appartenance à l'un ou l'autre Etat. Elle reprend simplement les limites des anciens kaza de Korçë et de Kastoria, qui séparent le bassin du Devoll du côté albanais et celui de l'Aliakmon du côté grec.

La région est alors habitée par des chrétiens orthodoxes et par des musulmans, sans frontière spatiale bien établie entre les deux communautés. Les chrétiens sont plutôt dans les villes, où ils s'occupent de commerce et d'artisanat, ainsi que dans les villages de montagnes, où ils vivent d'élevage et surtout grâce à l'émigration, saisonnière ou permanente, vers les villes ou vers l'étranger ; les musulmans, hormis les fonctionnaires ottomans, travaillent les terres agricoles, dont les propriétaires sont eux-mêmes musulmans. Par ailleurs, on ne peut distinguer formellement une population albanaise et une population grecque. Le multilinguisme semble répandu : on parle turc, grec, albanais, valaque, rom et macédonien - on disait alors "bulgare "- dans une imbrication à la fois sociale et spatiale, sans qu'il existe un lien nécessaire et exclusif entre une langue, une communauté religieuse et un territoire de quelque étendue. Les villes comportent toutes, par ailleurs, une population de juifs sépharades, artisans et commerçants, qui entreprennent après 1880 , comme les chrétiens, de racheter les terres musulmanes.

En dehors des milieux intellectuels et politiques, les allégeances nationales tardent à s'affirmer, surtout du côté albanais : le ralliement à la nouvelle Albanie est contesté à la fois par des musulmans soucieux de garder des liens avec l'empire ottoman et le sultan, et par des chrétiens considérant Constantinople comme la capitale de l'orthodoxie dans les Balkans. Ces gens sont stigmatisés, jusque dans l'historiographie communiste, sous les noms de turkoman et grekoman. On connaît l'anecdote, rapportée par Elie Kedourie, du villageois interrogé sur sa nationalité par la commission de délimitation de la frontière en 1913, et répondant en albanais "je suis grec" ${ }^{3}$, ce qui reflète la persistance des sentiments nationaux fondés sur le système du millet ottoman : les ethnonymes " grec", " turc" et " bulgare " renvoient ici aux communautés confessionnelles dirigées respectivement par le patriarche de Constantinople, le sultan et l'exarque bulgare. Le critère religieux reste en 1923 celui qui définit les échanges obligatoires, institués par le traité de Lausanne, entre musulmans de Grèce et chrétiens de Turquie. Aujourd'hui encore, les musulmans albanais du Devoll se désignent comme turcs, tout en se défendant d'appartenir à la nation turque; l'ethnonyme n'a ici qu'un sens religieux, il est synonyme de "musulman".

De chaque côté de la frontière, les États contribuent à développer le caractère national des populations; la frontière elle-même, obstacle à la

${ }^{3}$ Kedourie E. (1966), p. 124. 
communication et au maintien des relations quotidiennes qui fondaient les sentiments identitaires traditionnels - sur la base de l'appartenance religieuse, des réseaux de parenté, de la coutume commune ou de l'activité économique, participe à la formation d'un sentiment d'appartenance nationale chez les Grecs et les Albanais.

La Grèce a tôt fait d'entreprendre la purification ethnique de ses régions “ allogènes", c'est-à-dire en majorité non-grecques. En Épire, le partage de 1913 a laissé à l'Albanie la région hellénophone de Sarandë, et à la Grèce la partie de l'Epire que les Albanais appellent Çamëri et qu'ils revendiquent en 1912. Les Çams, ou Tourco-Tsamidès en grec, forment dans cette région une classe minoritaire de propriétaires terriens, albanophones et musulmans, face à la majorité chrétienne et hellénophone, composée surtout de métayers. Epargnés par les échanges de populations, les Çams sont néanmoins privés de leur suprématie sociale par la réforme agraire et le partage des terres, puis expulsés en 1944 sous l'accusation collective d'avoir collaboré avec l'occupant italien afin de récupérer les biens qui leur avaient été confisqués.

En Macédoine grecque, la purification est encore plus rapide et radicale. La frontière a laissé dans les nomes de Kastoria et de Florina non seulement de nombreux "Bulgares" (slavophones), mais aussi plusieurs villages de religion musulmane, comme Dipotamia (ex-Revan) ou Komninadès (Shag pour les Albanais). Inversement, plusieurs villages chrétiens, hellénophones ou vlachophones, sans compter l'élite marchande de villes comme Korçë, restent situés en territoire albanais. Après 1920, les échanges de population permettent à la Grèce d'expulser ou d'inciter au départ vers la Turquie quelque 416000 "Turcs et Bulgares " concentrés en Macédoine, en Thrace, dans les îles de l'Egée orientale et en Crète. Les terres libérées par les musulmans de Dipotamia sont attribuées par la Commission d'installation des réfugiés à quelques centaines de familles chrétiennes originaires de la région du Pont (Trébizonde et Sinop), qui y pratiquaient l'élevage. Leurs descendants, qui en rajoutent dans la grécité, jouent aujourd'hui sur le double sens du mot "turc" pour laisser entendre que les musulmans qui les ont précédés étaient des turcophones, façon de se prémunir de tout irrédentisme en niant toute ancienne présence albanaise, tant les arguments "historiques" conservent de poids dans le discours sur la légitimité des frontières. Il est vrai que de leur côté, avant l'ouverture de la frontière en 1991, les habitants du Devoll étaient persuadés que la région de Kastoria avait été peuplée d'albanophones jusqu'en $1924^{4}$.

Dans les nomes de Kastoria et de Florina, en 1913, la plus grande partie des "allogènes" sont cependant des slavophones, non concernés par les échanges

${ }^{4}$ De Rapper G. (1997). 
obligatoires $^{5}$, et dont l'effacement est plus progressif que celui des musulmans. L'interdiction de pratiquer leur langue et d'autres tracasseries incitent les "Slavo-macédoniens" à émigrer vers la Bulgarie ou les Etats-Unis dès les années qui suivent l'annexion. Pendant la guerre civile, leur combat au sein des troupes de l'EDES pour le rattachement de la Macédoine " égéenne " à la Macédoine yougoslave en fait des félons, que la Grèce, après 1949, prive de leurs droits civiques et expulse en nombre. Les Pontiques de Dipotamia se veulent au contraire d'un loyalisme sans faille : si les villages slavophones du Grammos sont vidés et parfois rasés, les villages de réfugiés conservent vie et activité. On ne peut se faire une idée précise du nombre de slavophones qui, aujourd'hui, persistent à s'identifier comme tels, tant la question reste tabou. Mais il est certain que la population de la Macédoine grecque est massivement attachée à la nation grecque, comme on a pu le voir en 1994 lors de la mobilisation contre la république de Macédoine, accusée d'usurper un nom "authentiquement grec" et d'avoir des visées expansionnistes sur la Macédoine grecque.

En 1923, les réfugiés transplantent à Dipotamia le système d'élevage polyvalent semi-transhumant, complété par des cultures spécialisées, dont ils vivaient sur les rives de la Mer Noire. Leurs bêtes disposent des pâtures communales qui dominent le village, ainsi que des récoltes de foin et de trèfle faites sur le lot de quelques dizaines de stremmata $^{8}$ que la réforme agraire attribue à chaque famille en usage individuel. On cultive aussi le tabac d'Orient, principale culture de rapport à laquelle l'installation des réfugiés donne une forte impulsion dans toute la Macédoine. Un tel système ne représente ni réelle rupture avec les pratiques antérieures, ni différence de fond avec celles du Devoll voisin. En Macédoine grecque comme en Albanie, les bas-fonds - lac Maliqi au nord de Korçë, Sarigol de Kozani ou rives de l'Axios - monopolisent longtemps les efforts de mise en valeur, laissant la montagne et les plateaux à l'écart des grandes transformations.

Du côté albanais, l'albanisation des "Bulgares" est déjà en route dans les années 1916-1920, à l'époque où Jacques Bourcart visite la région?. Dans le Devoll, les villages de Shuec, Rakickë et Zagradec, encore bulgares en 1918, sont aujourd'hui entièrement albanisés, même si les toponymes sont restés

\footnotetext{
5 La Bulgarie et la Grèce ont signé, en novembre 1919, une "convention d'émigration réciproque et volontaire " qui a permis d'encadrer une partie des déplacements entraînés par les guerres de 1912-1918. Mais en 1928 les slavophones représentent encore $30 \%$ de la population dans le nome de Kastoria, et $20 \%$ dans le nome de Florina.

${ }^{6}$ Selon la terminologie en usage dans le recensement grec de 1928.

${ }^{7}$ C'est-à-dire de Grèce, dans la terminologie slavo-macédonienne.

${ }^{8}$ Un stremma grec ou un dynym albanais égale 0,1 ha.

${ }^{9}$ Bourcart J. (1922), p. 274.
} 
slaves. Seul le village de Vërnik est officiellement rattaché à la minorité macédonienne qui peuple la rive sud-ouest du grand lac Prespa, et reçoit à ce titre un enseignement primaire en macédonien. La complète albanisation des chrétiens non-slavophones qui se désignaient et étaient désignés en 1913 comme " grecs " répond sans doute au même principe : laissés à l'écart de la minorité hellénophone d'Albanie du sud (districts de Delvinë, Sarandë et de Gjirokastër), ils n'ont pas eu le droit de recevoir un enseignement en grec, et répètent aujourd'hui à l'envi qu'ils sont "chrétiens, mais pas grecs": leur ralliement à la nation albanaise est total.

En Albanie comme en Grèce, la langue et l'enseignement ont joué un grand rôle, en particulier par l'interdiction d'utiliser ou d'apprendre la langue des voisins : jusqu'en 1991, il était interdit aux Albanais d'apprendre le grec ceux qui le faisaient en cachette étaient punis - et aujourd'hui encore l'enseignement du grec n'est pas prévu dans les collèges et les lycées de la région frontalière, alors même que les contacts et les échanges se multiplient entre les deux pays. De la même manière, l'albanais n'est pas une langue étrangère enseignée en Grèce. Un autre moyen de sceller l'appartenance des régions frontalières à la nation a été d'en changer la toponymie. Le procédé est largement utilisé en Grèce, où la plupart des toponymes slaves ont été hellénisés ou remplacés durant les années 1930: ainsi Revan, Shag, et Kostenec sont-ils devenus Dipotamia, Komninadès et Ieropiyi. Il est plus rare en Albanie, où plusieurs noms de villages du Devoll sont néanmoins remplacés en 1967: l'athéisme d'Etat ne supportant pas les noms à signification religieuse, qui pour certains étaient également slaves, Bozhigrad, "Villedieu", est ainsi devenu Miras, "Bonneville". Mais les anciens noms n'ont pas été oubliés, et leur utilisation prend aujourd'hui une dimension politique : préférer Mavrovo à Mavrochori, c'est rappeler l'origine slave de nombreux toponymes grecs et l'installation récente des habitants; insister sur l'emploi de Bozhigrad, c'est vouloir faire table rase de l'héritage communiste.

Au moment où la frontière est rouverte en 1991, elle met donc en contact deux populations aux identités nationales nettement affirmées. Elle a aussi gagné une dimension religieuse, en séparant l'Albanie musulmane et la Grèce chrétienne. Cette assimilation, au moins imaginée, entre nation et religion, pèse désormais sur les relations entre les deux populations, dans les régions frontalières comme ailleurs.

\section{Une frontière hermétique, des évolutions divergentes}

Les trajectoires politiques, sociales et économiques de Dipotamia et de Miras divergent après la Seconde guerre mondiale. Pendant la guerre civile grecque, l'Albanie, dirigée par les communistes, a servi de base arrière aux 
"partisans" repliés sur le Grammos; ils s'y réfugient après leur défaite ${ }^{10}$. Elle est en froid avec la Yougoslavie de Tito et en guerre avec la Grèce, et se retranche de ses deux voisins. La frontière avec la Grèce est fermée dès 1944. Elle devient étanche sur toute sa longueur après 1964, à la suite de la rupture entre l'Albanie et l'Union soviétique (1960-1961), avec la construction de la "clôture" (klon) : entre Miras et Dipotamia court une bande de terrain dégagée sur une dizaine de mètres de large, renforcée par une barrière électrifiée, et truffée de postes de surveillance d'où l'on tire sur les fuyards. Officiellement, il s'agit d'empêcher les infiltrations extérieures, alors que l'état de guerre perdure entre la Grèce et l'Albanie. Plus dissuasive encore, l'organisation de la population par brigades de quelques personnes où chacun est tenu pour responsable de la présence de l'autre.

Le village de Miras est alors complètement coupé de ses voisins grecs. Comme le racontent aujourd'hui encore les villageois, il suffisait de regarder trop ouvertement la frontière ou d'en parler pour être envoyé en prison. Miras n'est pourtant pas le plus mal loti des villages du Devoll : situé sur la rive gauche du cours d'eau, il n'est pas classé de façon permanente en zone frontière ; ses habitants peuvent circuler plus librement que ceux du village voisin de Qytezë, plus haut dans la vallée, et ne subissent pas la situation d'enfermement de Vidohovë, retranché du reste du monde entre la ligne frontière et la clôture.

Derrière cette frontière hermétique s'opère la collectivisation des terres. Le pouvoir communiste commence d'abord à distribuer la terre aux paysans (loi sur la réforme agraire d'août 1945), conformément au programme qui lui a assuré leur soutien. Mais en 1951 naissent les premières coopératives, simples rassemblements volontaires de quelques paysans. Sous la pression de l'Etat, le système collectiviste prend un caractère obligatoire et s'étend rapidement ; la collectivisation est terminée dans le Devoll dès 1956, alors qu'il faut attendre 1968 dans certaines régions du nord de l'Albanie ${ }^{11}$. Miras devient ainsi le centre d'une vaste coopérative regroupant les douze villages du haut Devoll et classée comme " collino-montagneuse" (kodrino-malore). Les coopératives sont un moyen de planifier la production, mais aussi d'embrigader la population, de limiter ses déplacements et de.contrôler les esprits.

\footnotetext{
${ }^{10}$ Considérés par Enver Hoxha comme des partisans de Tito, avec lequel il est brouillé, ces fuyards du Grammos seront aussitôt livrés à Staline qui leur fera purger de longues peines de camp ou de résidence forcée en Asie centrale.

11 Dans l'ensemble de l'Albanie, les surfaces exploitées en propriété privée représentent $91,4 \%$ de la SAU totale en $1950,13,6 \%$ en 1980 , et $2,7 \%$ en 1989. Parallèlement, le secteur d'Etat s'adjuge une part croissante des terres collectivisées - un quart de la SAU en 1990 par le biais des fermes dEtat et des coopératives de type "supérieur" au sein desquelles étaient incitées à fusionner les coopératives villageoises.
} 
Miras se spécialise dans les cultures et abandonne l'élevage, qui occupait la population des villages de montagne jusqu'à la deuxième guerre mondiale. Les troupeaux de moutons disparaissent et les vaches sont regroupées dans le troupeau collectif, sans produire suffisamment de lait pour toute la coopérative. La situation s'aggrave au début des années 1980, quand les mots d'ordre nationaux réduisent le lopin individuel des coopérateurs de 1000 à 300 mètres carrés, et interdisent la possession individuelle de tout bétail. De telles mesures exaspèrent les paysans sans qu'on les assouplisse à temps pour désamorcer le mécontentement. En 1989, les témoignages révèlent la misère des campagnes albanaises, où la natalité, qui oscille entre 25 et $35 \%$ selon les régions, a entassé une population dont les deux tiers ont moins de vingt ans, désœuvrée, démobilisée par la décrépitude du matériel, sans exutoire.

Pendant ce temps, Dipotamia connaît une évolution bien différente ${ }^{12}$, marquée par une mobilité aussi intense que la réclusion dont souffre Miras est pesante. L'exiguïté des exploitations et la pauvreté des agriculteurs poussent une majorité à émigrer : si Dipotamia est épargné par les déplacements de populations qui vident les localités du Grammos à l'issue de la guerre civile, ses habitants ne résistent pas aux contrats de travail proposés par l'Allemagne entre 1955 et 1975 . Un tiers d'entre eux résident à Dusseldorf, Francfort ou Nuremberg vers 1970 , et encore $15 \%$ aujourd'hui, tandis que d'autres familles se trouvent à Chicago ou en Australie. Encore cette estimation est-elle fondée sur l'examen du registre de citoyenneté local, où n'apparaissent que les ménages entretenant leurs attaches géographiques.

A l'émigration s'ajoute un exode rural intense vers Kastoria et Salonique. En 1960, la Grèce cherche à développer ses confins septentrionaux : la ville de Kastoria devient zone franche pour le commerce des déchets de fourrure, ce qui incite les fourreurs occidentaux à y implanter une sous-traitance très compétitive. En quelques années, la confection des vêtements en fourrures de récupération crée plusieurs milliers d'ateliers, qui font travailler vers 1980 près de 15000 personnes - les deux tiers de la population active du nomos quelques mois au moins dans l'année. A cette époque, Dipotamia compte 150 ouvriers ou artisans-fourreurs installés au chef-lieu avec leur famille proche.

Bien que ces petits fourreurs demeurent nominalement agriculteurs afin de bénéficier d'un régime fiscal avantageux, l'agriculture ne pèse pas lourd dans leurs revenus. Le travail de la fourrure offre en deux mois le gain d'un an sur 50 stremmata de blé. Artisans, ouvriers et émigrés confient donc leur terre en location à un parent, d'où la rapide concentration effective des terres au profit de quelques éleveurs professionnels qui réunissent 50 à 60 ha en moyenne, dix fois leur lot propre. La surface cultivée s'accroît aussi par le défrichement

12 Deslondes O. (1997), pp. 182-187. 
d'une grande partie des communaux après 1970. L'augmentation des surfaces cultivables et la diminution de la main-d'œuvre agricole supposent une simplification du système de production: les cultures spécialisées disparaissent, tabac en premier. Les efforts s'orientent vers un élevage plus intensif, tourné vers la production d'agneaux mais aussi de lait de vache, destinés au marché national. Les cultures fourragères comme le blé tendre et le trèfle occupent $90 \%$ des terres cultivées vers 1990 .

Depuis 1980, l'arrêt du recrutement de main-d'œuvre étrangère en Allemagne et les retours d'émigration ont enrayé le déclin de la population. Simultanément, une récession des ventes de fourrures a limité la période de travail à quelques semaines dans l'année, et contraint les employeurs à plafonner les rétributions pour rester compétitifs. De nombreux artisans et ouvriers précédemment installés à Kastoria se sont donc repliés sur l'élevage, qu'ils mènent de front avec un travail de la fourrure moins lucratif qu'auparavant. La pluriactivité a rendu à Dipotamia une certaine animation, mais l'ancienne société égalitaire a vécu: en 1992, le tiers des éleveurs détiennent les trois quarts du troupeau, et continuent de monopoliser les surfaces cultivables, parce qu'ils sont les seuls à être outillés en conséquence, et que le calendrier des travaux agricoles, de juin à décembre, concurrence celui de la fourrure. Pour la majorité des paysans, l'agriculture n'est qu'un appoint en complément d'une saison de travail plus ou moins brève comme fourreur.

L'aide financière de l'Europe, depuis 1981, a accéléré ces mutations et contribué à creuser l'abyssale disparité de revenus avec l'Albanie. Les subventions des fonds structurels ont permis aux agriculteurs de s'équiper en tracteurs puissants, en bergeries ou en matériel d'irrigation, et à la collectivité locale de financer d'importants travaux : en fusionnant au sein du dème d'Akritas comme l'y incitait la phase expérimentale de la réforme administrative aujourd'hui en cours d'application, les communes de Dipotamia, Komninadès et Chionato ont bénéficié dès 1992 d'une dotation majorée que le dème a utilisée pour asphalter la voirie, se doter d'un réseau d'adduction d'eau et d'assainissement, rénover les lieux publics. Le démarque $^{13}$ rumine à présent de vastes projets de développement agritouristique auxquels il aimerait associer ses homologues albanais : accueil chez l'habitant, xenonès ${ }^{14}$ d'altitude, sentiers à thèmes, etc. Bref, Dipotamia serait aujourd'hui un havre de paix si l'ouverture de la frontière, en apportant l'insécurité, n'était venue assombrir cette quiétude.

\footnotetext{
${ }^{13}$ Président du dème.

14 Auberge communale.
} 
Depuis 1990, une frontière-passoire

Avec la fin de l'état de guerre entre la Grèce et l'Albanie, en 1987, la frontière est entrouverte, et dans un seul sens : un poste de douane est installé à Kapshticë - "ce n'était qu'une table au milieu de la route" se souvient un ancien douanier - et des visiteurs venus de Grèce peuvent entrer en Albanie. Dans l'autre sens, des "fuyards" parviennent de temps à autre à passer en Grèce, mais le passage illégal de la frontière est toujours considéré comme une trahison envers la patrie, et il n'est pas encore question d'autoriser les passages légaux. Dans les villages proches de la frontière, on se souvient encore des tentatives, souvent désespérées, de tous ceux qui ont cherché à partir malgré la "clôture".

Pendant l'hiver 1990-1991, alors qu'à Tirana le pouvoir a déjà été ébranlé par la " crise des ambassades" de juillet $1990^{15}$, certains habitants du Devoll, jeunes hommes sans travail ou mal vus du pouvoir, commencent cependant à franchir la frontière pour se rendre en Grèce. Ils reçoivent un choc culturel intense et se retrouvent souvent parqués dans les casernes de Kastoria avant d'être ramenés en Albanie, mais les vêtements donnés par les Grecs et les récits ne font qu'encourager de nouveaux départs. Dans les villages les plus proches de la frontière, à Miras, à Vidohovë, à Qytezë, les gens se rendent en groupe dans les villages grecs pour y passer la journée, et principalement à Dipotamia, que beaucoup avaient fréquenté avant la guerre. Bien accueillis, nourris, ils reçoivent également des vêtements, mais leurs récits reflètent d'abord leur ahurissement devant le développement spectaculaire qu'a connu Dipotamia depuis la fin de la guerre : les rues asphaltées, l'éclairage public, les voitures, les maisons, tout y est différent. "On restait tous groupés, raconte aujourd'hui une villageoise albanaise, on avait peur de se perdre, de ne pas savoir comment revenir. Même pour aller aux toilettes, on y allait en groupe. On n'avait jamais vu ça ». La fête de Pâques de l'année 1991 reste dans beaucoup de mémoires : elle fut à la fois l'occasion de faire la fête " de l'autre côté " dans une ambiance de liberté - les gardes frontières grecs ne s'étaient pas opposés au passage des Albanais, les gardes albanais avaient disparu - et, pour les chrétiens comme pour les musulmans, elle marquait les retrouvailles avec la religion, réprimée en Albanie dès la fin de la guerre et totalement interdite depuis 1967. "Je m'en souviendrai toujours, raconte un villageois. A l'époque, nous n'avions pas encore de Coca ni de Fanta. Comme les bouteilles nous semblaient belles ! J'en avais ramené plusieurs, vides, et je les avais exposées sur le buffet de la salle. Maintenant, on en trouve partout

\footnotetext{
${ }^{15} 4500$ Albanais se réfugient alors dans les ambassades occidentales et demandent à quitter leur pays.
} 
et les gens les jettent dans la rue; repenser à tout cela maintenant nous fait sourire, mais ces bouteilles-là, je les ai toujours, elles sont au grenier ». C'est à cette époque que la clôture frontalière disparaît. Comme partout en Albanie, la population s'attaque à tous les symboles du pouvoir communiste : statues et monuments, mais aussi usines et bâtiments coopératifs. Laissés sans surveillance, les barbelés de la clôture sont recyclés par les villageois qui défendent leurs nouvelles parcelles privées. Les postes de garde disséminés dans la zone interdite sont pareillement abandonnés par l'Etat, et peu à peu détruits par les villageois en quête de matériaux de construction.

De leur côté, les Grecs insistent sur les secours qu'ils prodiguent aux " réfugiés ", et qui vont évoluer au gré des besoins avec l'appui des pouvoirs publics : à partir de 1992, l'Etat grec, la région de Macédoine occidentale et les municipalités de Kozani, Kastoria et Florina fournissent ainsi de vieux autobus, du matériel de soins, d'enseignement ou de télécommunication, cependant qu'une opération médiatisée permet aux paysans macédoniens de refiler leurs vieux tracteurs ${ }^{16}$ à ce qui reste de la ferme d'Etat du lac Maliq, dont les sols vierges de traitement chimique n'attendaient qu'un labour profond pour offrir des rendements atteignant 25 tonnes de betteraves sucrières à l'hectare.

Aide impuissante à endiguer le flot des immigrés clandestins : très vite, certains Albanais se voient proposer du travail et partent en Grèce pour plusieurs mois. Tout en pestant contre l'invasion, les Grecs - restaurateurs, agriculteurs, garagistes, maçons - leurs offrent par journée de travail près de la moitié du salaire mensuel d'un employé en Albanie, où les ratés de la transition et l'absence d'investissements retardent jusqu'à présent tout vrai démarrage économique : l'émigration s'impose comme une nécessité, avec quarante ans de retard sur la Grèce.

En effet, la décollectivisation des terres, entreprise dans les derniers temps du communisme, dès 1990 , avec la distribution de un à deux dynym ${ }^{17}$ par famille, répond à une exigence politique et sociale plus qu'aux nécessités économiques. C'est la “ loi pour le partage des terres" de juillet 1991, votée par le parlement issu des premières élections libres mais toujours dominé par les communistes, qui marque la fin des coopératives. La loi prévoit le partage égalitaire de la terre entre toutes les familles du village, en fonction du nombre de leurs membres. A Miras, chaque famille se retrouve à la tête d'une petite exploitation disposant de 1,74 hectare pour six personnes en moyenne, surface répartie en quatre endroits différents au moins selon les catégories de terre. Dans ces conditions, les parcelles de plus de 3000 mètres carrés sont

\footnotetext{
${ }_{17}$ Avant de racheter des engins neufs de forte puissance grâce aux subventions européennes.

17 Voir la note 9.
} 
rares, notamment dans les terrains de bonne qualité, c'est-à-dire irrigués, qui sont moins nombreux et donc plus fragmentés. Le morcellement parcellaire n'oblige pas seulement les gens à parcourir de longues distances d'un champ à l'autre; il interdit l'usage de moyens mécaniques. Comme les paysans n'ont pas encore de titres de propriété, la terre reste incessible et aucune forme de remembrement n'est possible.

L'agriculture est devenue familiale et le nouveau système de production a beaucoup changé. Vers 1980 , l'irrigation, à partir des réservoirs situés sur le piedmont de la Morava, permettait aux coopératives du Devoll de s'orienter vers les grandes cultures. Il y avait alors dans le bassin 8000 à 9000 ha de tabac, de betterave à sucre ou de blé sur 13000 ha cultivables. La disparition de l'organisation collective et étatique a entraîné celle des cultures industrielles, aujourd'hui remplacées par des cultures vivrières ou destinées à l'alimentation du bétail (légumes, maïs, trèfle), chaque maison entretenant une vache pour le lait. L'irrigation ne couvre plus que 4000 à 5000 ha car les réservoirs se sont envasés, les canaux sont hors d'usage ou pas assez ramifiés pour atteindre toutes les parcelles issues de la refonte agraire, les pompes sont en panne. La Direction de l'agriculture du district estime que près de 4000 hectares sont abandonnés, en particulier sur les collines, où l'irrigation n'est plus possible ${ }^{18}$.

Les cultures de rapport n'ont pas disparu pour autant : à Miras, on cultive toujours les oignons, comme on le faisait dans la coopérative. Mais si le savoir-faire et les rendements sont là - plus de 600 quintaux à l'hectare, voire 1000 les bonnes années -, les anciens réseaux de collecte et de distribution ont disparu avec la coopérative et les paysans écoulent leur production au coup par coup. Traumatisés par le collectivisme, ils répugnent à l'idée de se grouper pour vendre, et préfèrent s'en remettre au commerçant grec avisé qui leur imposera son prix. Mais les oignons grecs, qui arrivent plus tôt en saison, leur font concurrence. La récolte de l'année 1995, abondante, a pourri pendant l'hiver dans les fossés et les canaux d'irrigation de Miras, faute d'acheteurs.

Une agriculture aux revenus monétaires aussi incertains, et de loin incapable d'occuper tout le monde, voilà qui explique l'émigration. On estime que 7000 à 8000 personnes dans le Devoll séjournent régulièrement en Grèce pour une durée plus ou moins longue, soit $18 \%$ de la population totale, près d'une personne par famille. A Miras, la moitié des 4000 à 5000 personnes en âge de travailler - la quasi-totalité de la population masculine adulte - s'absente une partie de l'année, principalement en Grèce du Nord (Macédoine ou Thessalie). Les hommes quittent le village en mars-avril et

\footnotetext{
${ }^{18}$ Sur les conditions de la privatisation des terres dans le district de Korçë (qui englobait initialement le Devoll), voir Sivignon M. (1992).
} 
rentrent en septembre-octobre, laissant les femmes et les parents s'occuper des terres et de la maison.

Jusqu'en 1996, l'immigration albanaise et balkanique en Grèce est clandestine. Si, du côté albanais, aucun obstacle n'est mis au passage illégal de la frontière hors du poste frontière de Kapshticë, l'armée grecque surveille de son côté une large bande frontalière ; mais le caractère montagneux, boisé et souvent dépeuplé de la région laisse le champ libre aux clandestins, à condition de connaître le terrain. Les Albanais font ainsi la distinction entre passer "par la douane", c'est-à-dire par le poste frontière de Kapshticë, et "par la montagne". Certains habitants des villages frontaliers se sont spécialisés dans le rôle de passeurs à l'usage des clandestins originaires du reste de l'Albanie. Ils travaillent souvent en collaboration avec un passeur grec qui prend en charge les clandestins du côté grec, et les emmène là où ils veulent aller. Une telle prise en charge, d'Albanie jusqu'à Athènes ou Salonique, revient à 100000 drachmes par personne ${ }^{19}$. Comparativement, un passage par la "douane" avec un faux visa, réalisé par un faussaire albanais, coûte le double et le succès n'est pas assuré. Le résultat est qu'au premier janvier 1996, la police grecque des étrangers n'avait délivré en tout et pour tout que 4941 permis de séjour à des Albanais, soit 3,2\% des étrangers en situation régulière sur le sol grec : ce qui permettait aux journaux grecs de laisser courir les estimations les plus fantaisistes sur le nombre réel d'Albanais présents sur le sol grec, 500000 ou plus. L'émigration ne pouvait être estimée valablement que depuis l'Albanie, dont la population avait diminué de 60000 personnes en trois ans (1990-1992), malgré une croissance naturelle encore forte, estimée dans le même temps à 120000 , soit une émigration apparente de 180000 personnes, en Grèce pour la plupart. En 1996, 300000 Albanais - 10\% de la population albanaise - pouvaient simultanément se trouver en Grèce. Dans les régions de Fier ou de Korçë, des sondages confirmaient cette estimation ${ }^{20}$.

La situation précaire des immigrés albanais a permis aux autorités grecques de les utiliser comme moyen de pression sur Tirana, plusieurs fois sommée de se plier aux exigences d'Athènes au sujet de la minorité hellénophone d'Epire du Nord. Des rafles ont reconduit aux frontières 40000 clandestins en 1993, puis 70000 en 1994 en riposte au procès de cinq " séparatistes" membres de l'organisation vorio-épirote Omonia. Depuis cet épisode, les Grecs surveillent leur frontière et leur territoire d'une façon qui se veut impitoyable, tout en entreprenant depuis 1997 le recensement et la régularisation des étrangers. Le succès massif de cette opération témoigne aujourd'hui du degré de

${ }^{19} 1$ drachme égale 0,02 FF en 1998.
${ }^{20}$ Deslondes O., Sivignon M. (1995). 
normalisation auquel semble parvenir l'immigration albanaise en Grèce après des années de tensions ${ }^{21}$. L'immigré albanais et son patron grec ont établi au fil des ans des rapports stables et confiants, fortement paternalistes. Tout n'est pas réglé pour autant : d'après un récent sondage $e^{22}$, la moitié des Grecs souhaitent le départ de tous les étrangers, encore qualifiés de " réfugiés"; les employeurs grecs recrutent au coup par coup ; à Bilisht, on déplore l'absence d'une officine centralisant offres et demandes d'emploi.

Au fond d'un vallon autrefois désert, le passage frontalier de Kristallopiyi reflète cette évolution. Un large ruban de bitume neuf conduit au poste frontière grec, bâtiment modeste face au gros édifice décrépi où logent policiers et douaniers albanais, à cent mètres de là. Au-delà, vers Kapshticë et Bilisht, la route, étroite et bosselée, attend sa réfection. Septembre 1997 : cinq ou six camions immatriculés en Albanie attendent côté grec ; côté albanais, un bus et quelques taxis déposent des candidats au visa. En mal d'interlocuteur, éconduits par les policiers grecs, nous engageons la conversation avec l'un des chauffeurs albanais : Engjel est chrétien, son patron et lui sont de Korçë, il a installé sa famille dans la banlieue nord d'Athènes et semble bien intégré en Grèce. Arrivent deux autobus. Il en sort une centaine de clandestins reconduits à la frontière et qui gagnent à pied, sous bonne escorte policière, le poste albanais : scène fréquente et ordinaire. "Inutile de risquer sa peau et son argent dans la montagne, commente Engjel, mieux vaut tenter la légalité ". L'année suivante, à la même époque et au même endroit, les retours forcés auront disparu.

En marge de cette émigration au long cours existent des navettes quotidiennes de travailleurs frontaliers entre les villages du Devoll et leurs voisins grecs. Les habitants de Vidohovë, dans la commune de Miras, sont une majorité à se contenter de ces navettes qui les dispensent d'émigrer. Ils bénéficieront bientôt d'un statut particulier, en cours de négociation; en attendant, ils sont autorisés à entrer en Grèce pour la journée sur présentation d'un certificat d'emploi et d'une carte d'identité attestant leur lieu de résidence. Dipotamia emploie ainsi chaque jour, à la belle saison, une cinquantaine de personnes du Devoll comme bergers, bûcherons ou maçons. Les bergers gagnent 4000 drachmes par jour et sont nourris, les maçons jusqu'à 7000 drachmes. Ils gagneraient plus à Athènes, mais $y$ dépenseraient aussi beaucoup plus. Bref, le travail frontalier est une bonne affaire pour tous. A Dipotamia, on ne nie pas que le village serait moins prospère sans la maind'œuvre albanaise.

${ }^{21}$ En mars 1998 avaient été traitées 150000 demandes, dont 42,6\% d'Albanais.

22 Cité par Sotiris Vandoros (1999), Les réfugiés en Grèce : de l'exclusion sociale à la légitimation politique?, Helsinki Citizens's Assembly. 
Ce qui est gagné à l'étranger est surtout consacré à équiper sa maison réfrigérateur et téléviseur, puis chauffe-eau électrique et machine à laver - ou à s'en faire construire une neuve : la périphérie de Bilisht n'est qu'un vaste chantier où les constructions récentes ont précédé toute velléité de planification et d'équipement collectif, et la même fièvre s'empare de certains villages. Les gens ne croient plus aux placements mirobolants, échaudés par l'évaporation subite d'une société pyramidale de Pogradeç qui récoltait l'argent des émigrés " pour financer des travaux publics ", assurait-elle.

En dehors du bâtiment et de la maçonnerie, les investissements productifs semblent marginaux. Miras ne compte qu'une quarantaine de tracteurs, un pour 50 familles, et personne ne songe à un tel achat pour un ou deux hectares à cultiver. Quant à acheter du matériel en commun, l'idée fait encore frémir. La solution passera sans doute, comme c'est le cas en Grèce, par des arrangements individuels entre petits propriétaires et cultivateurs outillés, spécialisés dans le travail agricole. Les Albanais investissent encore moins d'argent dans l'artisanat et la production manufacturière, laissant aux Grecs toute l'initiative dans ce domaine. Ces derniers ont monté dans le Devoll cinq ateliers de confection; l'un d'eux occupe l'ancienne manufacture de tabac de Bilisht, et emploie de façon tournante 600 ouvriers dont beaucoup viennent chaque jour des environs. Les salaires locaux sont en effet dérisoires pour ce genre de tâche, 120 leks par jour $(5 \mathrm{~F})$ contre 4000 drachmes $(80 \mathrm{~F})$ en Grèce. Il s'agit pour les Grecs de tirer parti de cet énorme écart salarial sans beaucoup s'éloigner de leur territoire, dans un district resté par ailleurs à l'abri des émeutes et des saccages qui, en 1997, ont fait fuir les investisseurs grecs installés à Elbasan ou à Korçë.

\section{L'insécurité, obstacle au développement}

L'existence de relations hors-normes et les incidents qu'elles provoquent contribuent à entretenir, des deux côtés de la frontière, un sentiment d'insécurité qui pèse sur les possibilités d'échanges et de développement.

On peut regrouper sous la catégorie de contrebande une grande variété de trafics réalisés en dehors de la douane. Tout est susceptible d'être passé par la montagne, dans un sens comme dans l'autre, depuis les voitures volées en Grèce et emmenées en Albanie jusqu'aux napperons de dentelle réalisés par les Albanaises sous contrat avec des Grecs, qui fournissent le matériel et revendent en Grèce, en passant par le cannabis cultivé par les villageois albanais. Le passage des marchandises de plus forte valeur (voitures, drogue, cigarettes, matériel hi-fi, bétail) est aux mains de filières organisées pour lesquelles les villages frontaliers ne sont que des relais. Les autres échanges (sucre, paille, petit mobilier, laine) se font de manière individuelle, sur la base 
des relations que chacun peut avoir de l'autre côté. La contrebande de bétail, en particulier celle des agneaux dont les Grecs sont friands à Pâques, a longtemps été un des trafics les plus importants, impliquant des éleveurs de Korçë du côté albanais et, du côté grec, des bergers valaques de Iannina passant l'été dans le Grammos. Depuis 1995-1996, c'est une activité en perte de vitesse car les prix ont augmenté en Albanie, où un agneau valait 1500 drachmes en 1991 et 6000 à 8000 drachmes en 1996, et la contrebande n'est plus aussi rentable. La fièvre aphteuse a également obligé les autorités grecques à mieux contrôler la provenance de la viande entrant en Grèce.

Les frustrations nées des difficultés rencontrées en Grèce, plutôt qu'une tendance atavique au vol et à la destruction, poussent certains Albanais à tirer profit de la situation au dépens des Grecs, même si ces derniers ne sont pas les derniers à utiliser la jungle albanaise à des fins peu scrupuleuses : tel citoyen de Bilisht, qui s'était improvisé importateur de pêches de la région de Skidra, s'est fait abuser par son fournisseur grec en lui payant d'avance une cargaison qui ne lui est jamais parvenue. Mais de façon générale, ce sont les vols de matériel ou de bétail, "les prises d'otages" et les intimidations commis par les Albanais sur le sol grec qui enveniment régulièrement les relations, surtout, semble-t-il, depuis les pillages des dépôts d'armes lors des troubles du printemps 1997 en Albanie. "Chaque Albanais a au moins cinq armes ; ils ont même des bombes" prétend le démarque d'Akritas, qui raconte qu'au début de l'été 1997, des Albanais armés sont venus demander de l'argent à des moissonneurs grecs, et les ont emmenés en réclamant une rançon de cinq millions de drachmes. En allant discuter lui-même avec les ravisseurs, le démarque a obtenu la libération des cinq otages, sans rançon. Les "otages" sont parfois des vaches, des moutons ou des tracteurs que les Albanais acceptent de rendre contre rançon. De tels incidents alimentent à longueur de colonnes la presse de Kastoria, qui décrit la terreur où vivent chaque nuit les acritiques de Dipotamia et de Kristallopiyi.

Les réactions grecques sont de deux ordres. Après coup et pendant quelques jours, la police renforce la surveillance de la frontière et refoule les Albanais, y compris les journaliers de Miras et Vidohovë. D'autre part, les habitants de Dipotamia n'acceptent chez eux que les Albanais des villages les plus proches, qu'ils connaissent personnellement : " au moins, on sait où récupérer le bétail en cas de vol ". Le futur statut des frontaliers va dans ce sens.

La conséquence la plus grave de ce climat d'insécurité est qu'il retarde ou compromet les affaires sérieuses - échanges commerciaux, investissements grecs en territoire albanais, coopération - dont le rétablissement de l'ordre en Albanie est le préalable : tant qu'ils seront à la merci de vols ou d'émeutes, bien peu de Grecs se risqueront à franchir la frontière, a fortiori à investir en territoire albanais. Sans sécurité, pas de développement. Le mot est lâché. 
Homme cultivé, passionné et de bonne volonté, le démarque d'Akritas se dit convaincu qu'une Albanie développée sera le meilleur trait d'union entre la Grèce et l'Europe ; que la libre circulation des hommes et des biens, facilitée par l'aménagement d'une zone franche internationale et d'une liaison transfrontalière directe par Ieropiyi et Kapshticë via le " pont de Berisha " - à ce jour seule réalisation du projet -, transformera définitivement le cul-de-sac ouest macédonien en région de passage, et ramènera le temps des affaires enterré en 1941. Il bouillonne de projets communs où les Albanais sont les producteurs, les Grecs les investisseurs et les commerçants : culture biologique des oignons de Miras avec le fumier de Dipotamia, culture et conditionnement du "thé des montagnes" (tsaï tou vounou), tourisme "zoophile" et pédestre dans le Grammos ou la Morava, etc.

Au printemps 1998, les édiles et les responsables des districts albanais et des nomes grecs frontaliers, de Florina à la Thesprotie et du Devoll à Sarandë, se sont rencontrés pour tenter de fédérer et de coordonner de tels projets. Ils sont tombés d'accord pour demander à leur gouvernement de légiférer sur le statut des frontaliers, ou encore la possibilité de coopérer directement, notamment dans le domaine de l'éducation. Mais ces initiatives locales trouvent peu de répondant à Tirana. Le chaos institutionnel et politique qui y règne depuis 1997 n'entraîne pas seulement l'insécurité. Il prive Athènes d'interlocuteurs crédibles en matière de coopération, et l'Europe en matière de développement régional.

En effet, l'Union Européenne ne souhaite pas déroger au principe de subsidiarité qui subordonne le financement de tout programme à une participation du bénéficiaire, qui s'élèverait pour l'Albanie à $10 \%$ des dépenses. Pour symbolique que soit une telle participation, l'Etat ou les districts seraient bien en peine de l'acquitter. La dotation versée par l'Etat au district du Devoll pour couvrir ses dépenses d'équipement, selon son président, est en chute libre : 200000 dollars par an de 1992 à 1996, 30000 dollars en 1997 et en 1998, soit 4 dollars par habitant; le clientélisme politique détournerait l'essentiel des dotations vers quelques districts bien placés. Quant aux dotations versées aux communes et aux municipalités, elles sont insignifiantes et ne couvrent que de maigres dépenses de fonctionnement. Le vide institutionnel explique qu'aucun programme de type "Interreg " n'ait pu voir le jour pour donner vie aux projets de développement transfrontalier : cela prive de tout fondement l'idée, souvent admise à Bilisht, que les Grecs détournent l'aide européenne. Sur ce point, le Devoll n'est victime que de luimême ou de l'Albanie.

En attendant la participation de l'UE, l'aide européenne parvient à l'Albanie par d'autres canaux, comme le "Fonds albanais de développement", qui court-circuite Tirana, au sein duquel la France et l'Italie sont " responsables" 
du Devoll et de Korçë. Ainsi ont été financés en 1997 le réseau d'assainissement de Bilisht ( 85000 dollars) et l'adduction d'eau potable dans plusieurs villages. En 1998, les financements de ce type se seraient élevés à 160000 dollars. A eux seuls, ils pèseraient donc cinq fois plus que les dépenses publiques albanaises. Mais les Albanais sont unanimes à dénoncer le détournement de cette aide par une multitude d'intermédiaires véreux.

Une dernière condition essentielle de développement est mal assurée : la confiance mutuelle. Le discours des Grecs sur les Albanais et des Albanais sur les Grecs reste encombré de clichés, de procès d'intention et d'incompréhension.

\section{Représentations et discours}

L'arrivée massive de réfugiés albanais au début des années 1990 s'est accompagnée en Grèce de la généralisation d'une image négative des Albanais, dont le trait principal est la violence. Cette image trouve un écho dans la représentation des Albanais transmise par l'historiographie grecque concernant la période ottomane : souvent engagés dans les rangs de l'armée et de la gendarmerie ottomane, les Albanais apparaissent comme les auteurs de pillages et de cruautés commis au profit de l'empire contre la nation grecque. Les différents tracés de la frontière nord de la Grèce ont également été marqués par d'incessantes incursions d'irréguliers semant la terreur, d'un côté comme de l'autre de la frontière. C'est pourquoi l'image que l'historiographie albanaise donne des Grecs est elle aussi marquée par l'histoire de la frontière et la violence : on y rappelle les violences commises par les andartès ${ }^{23}$ grecs contre les villages d'Epire du nord fidèles à l'Albanie.

Malgré ces représentations " traditionnelles ", il n'est pas possible de parler d'une haine ancestrale entre Grecs et Albanais, qui expliquerait la méfiance et la violence dans leurs relations actuelles. La façon dont la peur de l'autre est construite et instrumentalisée, tant dans les reportages alarmistes de la presse que dans le discours sécuritaire des partis politiques, ne constitue qu'un aspect des représentations réciproques. Entre Miras et Dipotamia, les représentations semblent plus nettement liées à la nature des échanges et des contacts entre les deux villages qu'à l'image traditionnelle de l'autre, même si celle-ci peut toujours être rappelée et utilisée. On peut d'ailleurs s'interroger sur l'importance de ces images traditionnelles pour une population qui n'est installée à Dipotamia que depuis 1924 , c'est-à-dire après les temps difficiles où Grecs et Albanais s'affrontaient pour le tracé de la frontière. De la même manière, l'image du Grec fourbe et cruel est souvent rejetée par les Albanais

23 "Irréguliers" (à l'époque ottomane), "rebelles" (pendant la guerre civile). 
d'aujourd'hui, pour lesquels elle est trop fortement associée à la propagande de l'époque communiste. Pour toutes ces raisons, il semble préférable de considérer ici les représentations et les discours dans leur contexte local, en fonction des relations entre les deux villages.

La longue période de fermeture de la frontière (1944-1991) et les évolutions divergentes qu'ont connues les deux villages ont renforcé l'idée d'une irréductible différence entre les deux populations. Dans les discours actuels, tout est bon pour affirmer la limite entre " nous" et " eux". Cet acharnement identitaire est d'autant plus fort qu'il existe en fait, malgré les divergences économiques et politiques, une indéniable proximité culturelle entre Grecs et Albanais. Dans ces conditions, une différence apparemment anodine peut être élevée au rang de valeur identitaire. C'est le cas, pour les Albanais, de l'alphabet. Les habitants de Miras qui vont travailler en Grèce ne cessent d'affimer que leur alphabet est supérieur à l'alphabet grec, non par la forme des lettres, mais par leur nombre : " les Grecs se prennent pour des donneurs de leçons, mais ils n'ont que 24 lettres; nous en avons 36 " dit l'un d'eux ${ }^{24}$. Cet exemple montre comment la différence affirmée entre Grecs et Albanais est teintée de hiérarchie : les deux populations ne sont pas à égalité et les Grecs sont, d'une manière générale, en position de supériorité. Cette supériorité est à la fois économique (ils sont plus riches), politique (ils ont un Etat stable et démocratique) et culturelle (ils sont Européens). Chacun conçoit l'avenir comme une progression des conditions de vie des Albanais vers l'état actuel des Grecs, même si l'inégalité doit subsister : les Albanais ne cessent de dire qu'ils sont en retard et que les Grecs sont en avance. Ces derniers ne pensent pas le contraire : quand on lui demande si l'augmentation des salaires en Albanie ne va pas rendre moins attractif le travail albanais en Grèce, le démarque d'Akritas répond : "les salaires albanais ne rejoindront pas les salaires grecs, même dans vingt ans. Parce que nous nous développons aussi ». Les Albanais de Miras continueront donc, à leur manière, à participer au développement de Dipotamia tout autant qu'à celui de leur propre village.

L'infériorité des Albanais se traduit d'abord dans les relations de travail. On a vu que leur survie passe par l'émigration vers la Grèce, où ils ne sont d'ailleurs jamais sûrs de pouvoir travailler le temps qu'ils souhaitent, à la merci d'une expulsion. Cette précarité maintient les migrants dans une position d'infériorité, sans autre solution que d'accepter les bas salaires et l'absence de couverture sociale. Un des motifs de frustration les plus fréquemment évoqués est l'humiliation ressentie par les Albanais diplômés employés par des patrons grecs sans instruction. C'est notamment le cas des

${ }^{24}$ Lusage albanais est, en effet, de compter comme "lettre" les groupes de consonnes à valeur phonétique particulière (sh, zh, xh, etc) et les lettres avec signes diacritiques (c, ë). 
enseignants qui vont travailler en Grèce le temps des vacances scolaires. Les Albanais ont ainsi le sentiment, parce qu'ils sont en position de faiblesse économique, d'être considérés comme inférieurs dans tous les domaines.

L'histoire de Riku, instituteur originaire de Miras, telle qu'il la raconte luimême, est révélatrice de cette situation : âgé d'une trentaine d'années, Riku a étudié les beaux-arts à Tirana avant d'être nommé instituteur dans une école de village à une heure de marche de chez lui, vers la frontière grecque. En 1991, il s'est aventuré avec d'autres instituteurs jusqu'à Dipotamia. A force d'y retourner, il a appris quelques mots de grec, et a pu discuter avec les gens qui le recevaient. "Que fais-tu là-bas en Albanie ? lui demandait-on. - Je suis peintre, répondait-il, je fais des tableaux. - Peintre ? Les Albanais sont-ils capables de peindre ? Comment pourraient-ils être artistes ? Tu nous racontes des histoires !». Comprenant que la plupart des Grecs considèrent les Albanais comme des ignorants sans culture, il revient à Dipotamia avec ses tableaux ; mais les Grecs refusent de croire qu'il les a faits lui-même. Il leur propose alors de réaliser un portrait, devant eux, et ils sont bien obligés de reconnaître qu'il y a des peintres en Albanie. De plus, son travail leur a plu et ils lui passent des commandes. Aujourd'hui, les murs du terrain de sport, de l'école, du poste de police et de plusieurs maisons particulières sont décorés de fresques du "peintre albanais", comme il se fait désormais connaître auprès des gardes frontière grecs. Musulman, il est aussi devenu spécialiste de l'icône chrétienne et trouve fréquemment du travail dans les églises de la région.

De leur côté, tout en reconnaissant la part des clandestins albanais dans l'essor de l'économie locale, les habitants de Dipotamia insistent sur l'aide et l'assistance qu'ils fournissent aux villages de l'autre côté, comme pour atténuer les profits tirés du travail clandestin. Quand le démarque évoque les projets de développement bilatéraux, son discours - "le pays est riche, la terre est fertile, mais ils ne travaillent pas, ils ne pensent qu'à voler et à se battre" - met l'accent sur une Albanie sous-développée et dépendante de la Grèce, que contestent les Albanais. L'aide apportée aux écoles du district ? “ Il ne s'agit que de matériel; ce n'est pas un problème de niveau, il est meilleur qu'en Grèce". Le don de matériel grec pour relancer la production de betterave sucrière ? «Nous ne sommes pas au courant, dit-on à la Direction de l'agriculture de Bilisht, mais les Grecs sont des menteurs ».

L'infériorité albanaise est également politique. A Miras comme à Dipotamia, on déplore l'instabilité et l'inefficacité de l'Etat albanais. La lutte entre le Parti socialiste, au pouvoir depuis les troubles de 1997, et le Parti démocratique de l'ancien président Sali Berisha, est suivie à Dipotamia de plus près que la situation au Kosovo, car on lui attribue une influence directe sur la région : à chaque crise politique (hiver 1996-1997, septembre 1998), 
les habitants de Dipotamia craignent à la fois un afflux de réfugiés et le développement de bandes armées profitant de l'effondrement de l'autorité centrale. Les Grecs parlent de "luttes de clans" pour évoquer les rivalités pour le pouvoir en Albanie, et ils voient en Sali Berisha, musulman et " homme du nord [de l'Albanie] ", le principal responsable de l'instabilité. Son adversaire Fatos Nano, originaire du sud et plus sensible à la proximité de la Grèce, apparaît au contraire comme un "modéré" (metriopathis). Clans, fanatisme, islam : l'image que les Grecs dressent de l'Etat albanais est tout le contraire de ce que l'on peut attendre d'une démocratie européenne, et la Grèce apparaît a contrario comme un pays stable et démocratique, dont l'appartenance à l'Union européenne est un gage de culture politique.

Le discours des Grecs de Dipotamia sur l'Etat albanais est d'ailleurs très proche de celui des gens de Miras, et du Devoll en général, sur le nord de l'Albanie: on y relève la même association de pauvreté, de violence, de clanisme et de fanatisme. Les gens du Devoll revendiquent au contraire un ensemble de traits " positifs ", l'éducation, la modération, l'ardeur au travail, qu'ils expriment par le mot "culture" et par lesquels ils se désolidarisent du nord du pays. Bien que la question de la "culture" dans le Devoll soit bien antérieure à la réouverture de la frontière, on ne peut que constater ici une convergence de vues entre Grecs et Albanais de la zone frontalière, comme si la coexistence quotidienne prenait le dessus sur les peurs et les représentations caricaturales : “ Ceux d'en face sont de braves types, répète-t-on à Dipotamia au sujet de Miras, on ne risque rien, même s'ils sont armés. Le problème, ce sont les mafieux qui viennent de plus loin ". A mesure que les échanges et les contacts se multiplient entre villages frontaliers, l'Autre qui fait peur recule un peu plus. Ce qu'un habitant de Bilisht résume d'un ton amusé : “ les Grecs ne vont pas au-delà de Korçë, ils ont peur !"

Les réactions albanaises à la position d'infériorité qui leur est reconnue et qu'ils se reconnaissent eux-mêmes sont de plusieurs sortes. Une première solution est de rechercher une supériorité à un autre niveau, différent du seul développement économique et politique. C'est le cas, on l'a vu; lorsque l'alphabet devient le marqueur d'une supériorité culturelle. Un des exemples les plus souvent avancés par les Albanais est celui des relations humaines. Les Grecs ont beau être plus riches et plus développés, disent-ils, ils ne sont pas aussi chaleureux, aussi accueillants et aussi droits que les Albanais : "ils n'ont pas d'amis et ne rient jamais ". En comparant la nature des relations familiales, les Albanais évoquent avec réprobation l'existence de maisons de retraite qui permettent aux enfants d'abandonner leurs parents âgés, alors qu'un tel comportement est impensable en Albanie.

Un autre type de réaction fait appel à l'histoire : les Albanais cherchent dans le passé un temps où les positions relatives des Grecs et des Albanais étaient 
inversées. Ils le trouvent avant la deuxième guerre mondiale, lorsque les Grecs d'Asie mineure sont arrivés dans les villages qu'ils occupent aujourd'hui. Les gens du Devoll racontent que ces réfugiés, démunis, venaient chercher pain et travail dans les villages albanais, alors plus prospères, ce qui leur permet d'affirmer que leur position actuelle n'est qu'un accident historique et qu'ils ont été, eux aussi, les bienfaiteurs de ceux qui se posent aujourd'hui comme leurs bienfaiteurs. Et ils ne sont pas loin de penser qu'une part des vols et des intimidations commis à Dipotamia sont le juste retour de l'exploitation qu'ils estiment subir de la part des Grecs : car si les Grecs perçoivent les Albanais comme des voleurs, ces derniers ont aussi le sentiment de se faire "voler" leur seule richesse, leur force de travail.

\section{Bibliographie}

Bourcart Jacques (1922), Les confins albanais administrés par la France (1916-1920). Contribution à la géographie et à la géologie de l'Albanie moyenne, Paris, Delagrave.

Danforth Loring M. (1993), "Claims to Macedonian Identity. The Macedonian Question and the Breakup of Yugoslavia", Anthropology Today, vol. 9 (4), 3-10.

De Rapper Gilles (1996), "Frontière et transition en Albanie du sud". Europaea, II, 1, 65-75.

De Rapper Gilles (1997), “Les villages de Kastoria ou la minorité imaginée ", Synchrona Themata, $\mathrm{n}^{\circ}$ 63, avril-juin 1997, Athènes, 108-111 [en grec].

De Rapper Gilles (1998), La frontière albanaise. Famille, société et identité collective en Albanie du Sud, thèse de doctorat, Nanterre.

De Rapper Gilles, Deslondes Olivier, Roux Michel (1998), "Les Albanais hors d'Albanie : émigrés et minorités en Macédoine, en Grèce et au Kosovo ", Hérodote, 90, 20-45.

Deslondes Olivier (1997), Les fourreurs de Kastoria, entre la Macédoine et l'Occident, Paris, CNRS Editions.

Deslondes Olivier, Sivignon Michel (1995), "L'agriculture albanaise, de la coopérative à l'exploitation de survie" in Décollectivisations agraires en Europe centrale et orientale, Revue d'Études comparatives Est-Ouest, vol. 26, n³, 143-160.

Gut Agnès (1979). Les frontières de l'Albanie, Thèse de troisième cycle, Université de Paris III - INALCO, Paris.

Kedourie Elie (1966), Nationalism, Londres, Hutchinson.

Puto Arben (1977), "Qeveria demokratike e 1924-s për zgjidhjen e problemeve të mbetura pezull në caktimin e kufijve të Shqipërisë [le 
gouvernement de 1924 et la solution des problèmes restés en suspens dans la délimitation des frontières de l'Albanie]" in Cështje të lëvizjes demokratike dhe revolucionare shqiptare në vitet 1921-1924, Akademia e shkencave e RPSSH, Tirana, 173-199.

Vickers Miranda, Pettifer James (1997), Albania. From Anarchy to a Balkan Identity, Londres, Hurst.

Sivignon Michel (1992), “La nouvelle agriculture en Albanie et l'exemple de Korçë ", L'Europe entre deux modèles agricoles, Revue géographique des Pyrénées et du Sud-Ouest, t. 63, fasc. 2, pp. 289-301, Toulouse.

Wilkinson H. R. (1951), Maps and Politics. A Review of the Ethnographic Cartography of Macedonia. Liverpool, University Press. 
F ig 1 - La rógion frontalière de Kastoria (Grèce) ot de Bilisht (Albanie)

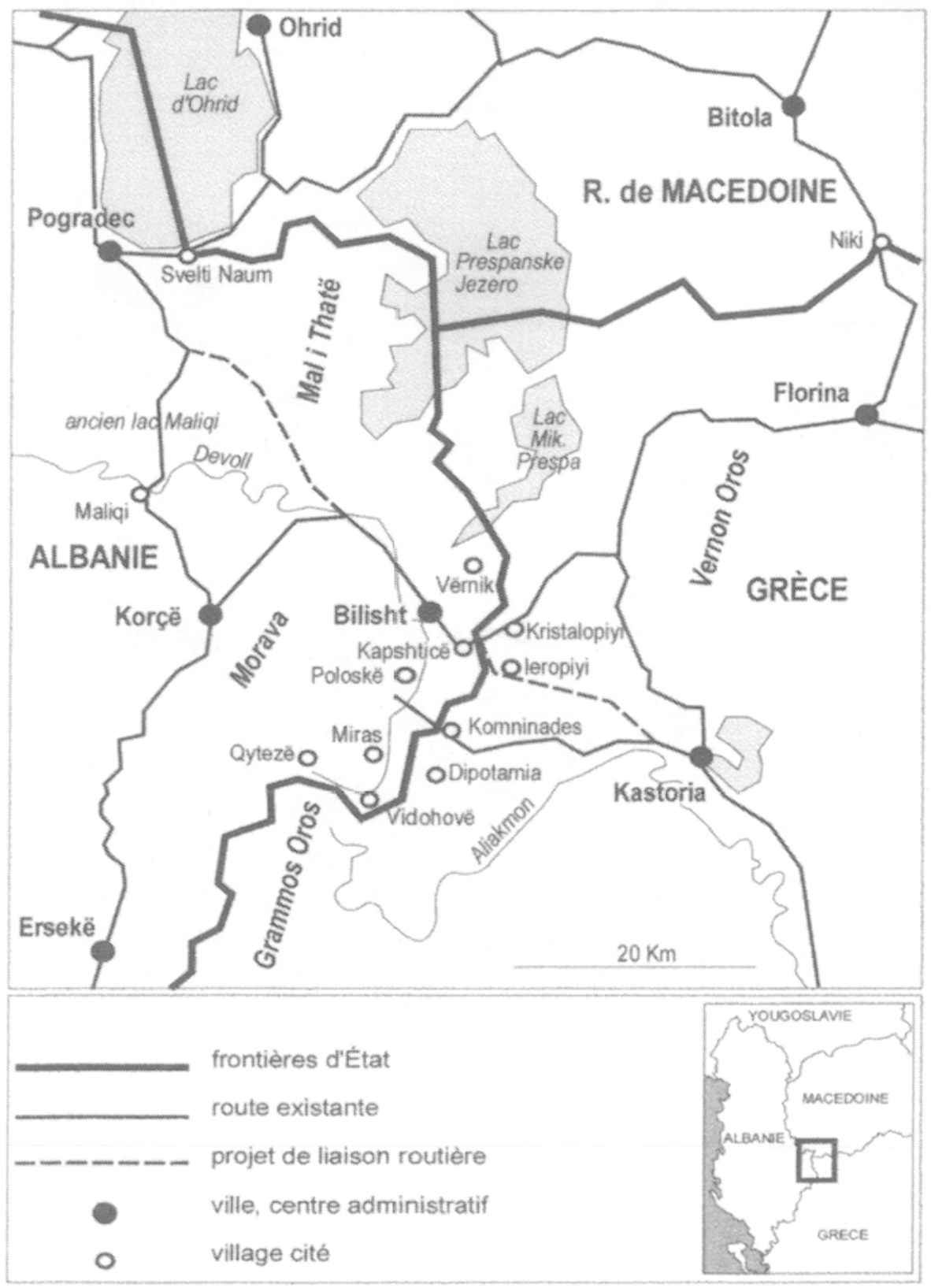




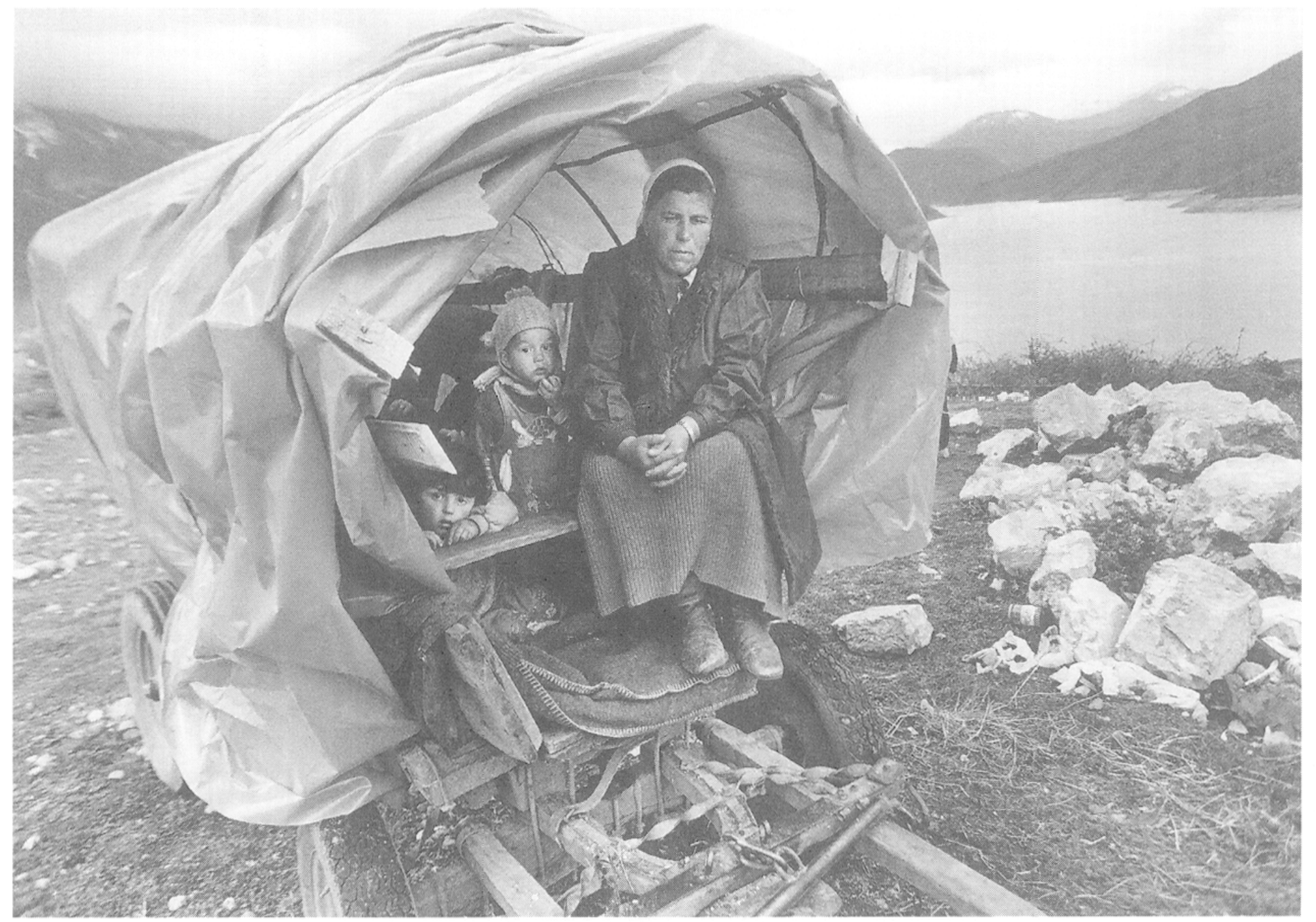

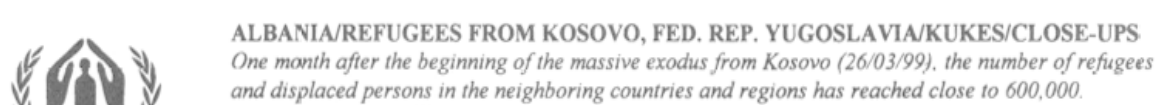

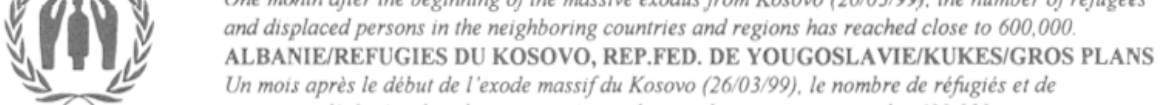

$$
\begin{aligned}
& \text { UNHCR } \begin{array}{l}
\text { personnes deplacees dans les pays et régions limitrophes atteint presque les } 600000 . \\
\text { Photo: UNHCR/29026/04.1999/R. Chalasani }
\end{array}
\end{aligned}
$$

